\title{
DESARROLLO LÓGICO MATEMÁTICO UNA MIRADA DESDE LA FONOAUDIOLOGÍA
}

\section{Logical mathematical developmenta look from fonoaudiology}

Martínez M Aura J(1), García Luisa $\mathrm{F}^{(2)}$, Jaimes Enna B(3), Vivas Angely, Meneses María A, Guerrero Natalia

\section{RESUMEN}

INTRODUCCIÓN: En la presente investigación se pretende determinar la adquisición y desarrollo del proceso Lógico Matemático en niños de 0-7 años de edad, identificando habilidades que permiten resolver operaciones básicas, analizar información, hacer uso del pensamiento reflexivo y del conocimiento del mundo para aplicarlo a la vida cotidiana, haciendo un recorrido en la historia académica, teórica y terapéutica sobre el desarrollo, la estimulación, los procesos y eventos que favorecen el desarrollo lógico y que determinan su importancia en la vida del ser. MÉTODOS: se desarrolla a partir de una Revisión sistemática Cochrane, acerca del desarrollo y adquisición del proceso Lógico Matemático y las pruebas de evaluación más utilizadas en estas edades. Se realizó la búsqueda en bases de datos científicas y no científicas y en fuentes adicionales de ensayos publicados. RESULTADOS: Se encontró un total de 33.763 estudios de acuerdo a los criterios establecidos, de los cuales 23 fueron incluidos. Se realizó un inventario de las metodologías disponibles, describiendo sus propiedades, aspectos a evaluar y edades de aplicación. ANÁLISIS Y DISCUSIÓN: Las matemáticas son una actividad cultural social e históricamente situada, influenciada por criterios prácticos de utilidad e intencionalidad y basada en prácticas cotidianas como contar, medir, localizar, diseñar, jugar o explicar. CONCLUSIONES: De acuerdo al enfoque piagetiano el mundo físico y social en el que se desarrollan los niños pequeños les brinda la oportunidad de adquirir numerosos conceptos relacionados con la aritmética, antes de la enseñanza formal.

PALABRAS CALVE: Desarrollo, lógica, matemática, fonoaudiología, aprendizaje, evaluación.

\begin{abstract}
INTRODUCTION: The present research aims to determine the acquisition and development of the Logical Mathematical process in children 0-7 years old, identifying skills that allow solving basic operations, analyze information, make use of thought and knowledge of the world to apply it To the daily life, making a journey in the academic, theoretical and therapeutic history on the development, the stimulation, the processes and events that favor the logical development and that determine its importance in the life of the being. METHODS: it is developed from a Cochrane Systematic Review about the development and acquisition of the Logical Mathematical process and the evaluation tests most used in these ages. We searched scientific and non-scientific databases and additional sources of published trials. RESULTS: A total of 33,763 studies were found according to established criteria, of which 23 were included. An inventory was made of the available methodologies, describing their properties, aspects to be evaluated and ages of application. ANALYSIS AND DISCUSSION: Mathematics is a social and historically situated cultural activity, influenced by practical criteria of utility and intentionality and based on daily practices such as counting, measuring, locating, designing, playing or explaining. CONCLUSIONS: According to the Piagetian approach, the physical and social world in which young children develop gives them the opportunity to acquire numerous concepts related to arithmetic, before formal education.
\end{abstract}

(1) Fonoaudióloga. Especialista en Pedagogía Universitaria. Magister en Intervención Educativa y Psicológica (2) Fonoaudióloga. Cadidata a Doctorado en Educación (3) Fonoaudióloga. Especialista en Audiología. Especialista en Seguridad y Salud en elTrabajo. 


\section{INTRODUCCIÓN}

El desarrollo del pensamiento matemático temprano (Primera infancia, de 0 a 6 años), pone las bases para el ulterior desarrollo matemático del niño, y aunque resulta poco frecuente que en él se detecten dificultades de aprendizaje, es sabido que un desarrollo defectuoso durante esta fase contribuye, a la aparición de problemas posteriormente de la misma manera que una buena estimulación reducirá el riesgo de su aparición.

Es por ello la necesidad de conocer y analizar los modelos evolutivos para adquirir el proceso matemático en su normalidad y en las diferentes patologías neurológicas; con el fin de determinar las dificultades que pueden presentarse por una inadecuada estimulación.

Es frecuente que en las escuelas no se detecten las dificultades de aprendizaje de los procesos o pensamiento matemático antes de $3^{\circ}$ de primaria, y no es posible diagnosticar con cierto rigor su existencia antes de los 6 años. Sin embargo, sí es habitual que los niños no tengan el mismo ritmo de aprendizaje, que algunos experimenten retrasos y encuentren más dificultades que otros para adquirir algunos conocimientos. La atención a ciertos indicadores de riesgo es útil para prevenir y corregir algunas complicaciones antes que lleguen a constituir problemas más serios y difíciles de corregir.

Los procesos Logicomatemático inician en el mundo desde la misma necesidad de la humanidad, por sobrevivir, por apearse de alimento y objetos para su subsistencia, es así que desde la prehistoria el hombre inicia con procesos de asociación relacionados con el tiempo, para determinar los periodos de sequía y abundancia, más adelante y cuando la necesidad lo hizo sedentario, se vio en la obligación de contar, agrupar, distribuir y muchos otros procesos lógicos, con el fin de tener alimento, animales, vegetación que cubriera sus necesidades, es así en el desarrollo y evolución de la humanidad misma, que el hombre se ve obligado a desarrollar sus habilidades lógicas, desde allí muchos autores como Gelman y Gallistel (1978), Fuson (1988) y Bermejo, Lago y Rodríguez (1989), inician la investigación sobre esta área del saber y llegan a conclusiones que hasta difieren a la hora de explicar la forma en que los niños obtienen una representación de la cantidad numérica y del papel que los procesos perceptivos juegan en la misma.

La adquisición de competencias necesarias para que se produzcan la comprensión del número está condicionada por el desarrollo previo, como los son los procesos cognitivos básicos (percepción, memoria, atención, razonamiento); conceptos básicos (forma, tamaño, cantidad, orden, posición), conocimiento de símbolos y signos necesarios para operar.

Es muy importante y básico que en la primera infancia todos los niños aprenda a ser lógicos (Nunes y Bryant, 2005). Es así que solo aquellos sujetos que aprendan las reglas de la lógica podrán entender y realizar adecuadamente las tareas matemáticas más simples y elementales. Por tanto es necesario reconocer a la lógica como uno de los constituyentes del sistema cognitivo de todo sujeto (Chamorro, 2005). Su jerarquía es que permite instaurar las bases del razonamiento, así como la construcción no solo de los conocimientos matemáticos sino de cualquier otro perteneciente a otras asignaturas del plan de estudio.

Es por esto que surge la pregunta macro ¿A partir de qué momento se puede hablar del aprendizaje de las matemáticas? ¿De qué forma se logra identificarlas y diagnosticarlas?

\section{MÉTODOS}

Para el desarrollo del presente trabajo se implementó como metodología de investigación la revisión sistemática Cochrane de tipo metodológica, que tiene como objetivo reunir toda evidencia que corresponda con el cumplimiento de unos criterios elegidos y establecidos previamente (2), con el fin de orientar un tema específico de investigación, en primera instancia se debe dar a conocer la adquisición y el desarrollo del proceso Lógico Matemático en niños de 0-7 años de edad,

y su proceso de evaluación.

Rev. Signos Fónicos Vol $3 \mathrm{~N}^{\circ} 1$

Abril / Septiembre 2017 
Los criterios de exclusión que se tuvo en cuenta:

Las edades no comprendidas entre los 0 a 7 años, publicaciones inferiores a 10 años, que no fuesen estudios de revistas indexadas, estudios en animales.

Los criterios de inclusión en el estudio fueron:

Artículos referentes a los procesos matemáticos en edades comprendidas de 0 a 7 años y su desarrollo lógico matemático, teniendo en cuenta una línea de tiempo de 10 años, estudios en humanos dentro y fuera del país de origen.

Los ejes temáticos de los estudios incluidos, partieron del desarrollo del proceso lógico matemático en la primera infancia y la evaluación del nivel de desarrollo matemático en edades comprendidas entre los 0-7 años.

Se realizaron búsquedas en las bases de datos científicas como Redalyc, Dialnet, ScieLo, PubMed, Iresei, Science Direct[1] en otras bases de datos electrónicas y en fuentes adicionales de ensayos publicados (búsqueda más reciente: 10 de Mayo del 2017). Durante la revisión se consultaron bases de datos científicas, se seleccionaron los títulos y los resúmenes para la inclusión de forma independiente y se discutieron los resultados.

Los artículos revisados se categorizaron de acuerdo al tipo de instrumento, a saber: test; baterías de evaluación, check list y protocolos; Posteriormente se estableció el análisis de información publicada en una línea de tiempo de 10 años.

\section{RESULTADOS}

Los datos seleccionados están considerados como datos nominales (incluidas las escalas de medición) donde el resultado es una categorización, que genera un inventario de las aproximaciones conceptuales del desarrollo lógico matemático y sus posibles pruebas de evaluación. A partir de la búsqueda en las bases de datos se encuentran una cantidad determinada y considerable de artículos, permitiendo de acuerdo a las palabras clave establecidas, se encontró un número de publicaciones distribuidas como lo expone la Tabla 1.

Tabla 1. Distribución de los estudios encontrados de acuerdo a los criterios de búsqueda.

\begin{tabular}{cc}
\hline Fuente o Base de Datos & Número de Estudios \\
\hline Scielo & 3.560 \\
\hline Elsier & 10 \\
\hline Dialnet & 30.216 \\
\hline Redalyc & 22.242 \\
\hline Otras fuentes & 4 \\
\hline
\end{tabular}


En la tabla 1, se refleja a nivel general el total de estudios perteneciente a cada eje temático, siendo un total de 33.763 artículos en adquisición y desarrollo del proceso lógico matemático de los cuales 3.560 pertenecieron a ScieLo, 10 a Elsier, 30.164 a Dialnet y 29 en Redalyc. Por otra parte en la búsqueda de las pruebas de evaluación a nivel nacional e internacional se obtuvo un total de 52 pertenecieron a Dialnet, 22.213 a Redalyc y 4 correspondientes a otros estudios.

Tabla 2. Variables de búsqueda

\begin{tabular}{|c|c|c|}
\hline Variable Independiente & Variable Dependiente & Cruces de Variables \\
\hline Niños 0-7 años & $\begin{array}{l}\text { Learning } \\
\text { Logic } \\
\text { Mathematic } \\
\text { Cognition } \\
\text { Metacognition } \\
\text { Knowledge }\end{array}$ & $\begin{array}{l}\text { Child + development + logic }+ \\
\text { mathematic. } \\
\text { Metacognition + child + } \\
\text { development + logic }+ \\
\text { mathematic. } \\
\text { Knowledge + child + } \\
\text { development + logic + } \\
\text { mathematic. } \\
\text { Cognition + logic }+ \\
\text { mathematic + child }\end{array}$ \\
\hline Niños 0-7 años & $\begin{array}{l}\text { Evaluation } \\
\text { Mathematic } \\
\text { Logic } \\
\text { Mathematical development. } \\
\text { Cognitive process }\end{array}$ & $\begin{array}{l}\text { Evaluation + mathematic + } \\
\text { children. } \\
\text { Evaluation + mathematical } \\
\text { logistic development. } \\
\text { Evaluation + cognitive process } \\
+ \text { mathematical development. } \\
\text { Evaluation + mathematical } \\
\text { development + child. } \\
\text { Test + evaluation mathematic } \\
+ \text { child. }\end{array}$ \\
\hline
\end{tabular}

En la tabla 2, se evidencia las variables independientes y dependientes utilizadas en la revisión sistemática perteneciente a; Adquisición y desarrollo del proceso lógico matemático, pruebas de evaluación a nivel nacional e internacional.

En la tabla 3, se reporta el total de estudios incluidos en la revisión perteneciente a cada eje temático, en adquisición y desarrollo del proceso lógico matemático se utilizaron 5 artículos de la base de datos Dialnet, 4 de Redalyc, 1 de Elsier y 3 de Scielo; para las pruebas de evaluación a nivel nacional e internacional se emplearon 2 artículos hallados en Dialnet, 4 de Redalyc y 4 correspondientes a otros estudios; para un total de 23 estudios incluidos en este proceso investigativo. 
Tabla 3. Distribución de los estudios incluidos para la revisión

\begin{tabular}{lc}
\hline Fuente o Base de Datos & Número de Estudios \\
\hline Dialnet & 7 \\
\hline Redalyc & 8 \\
\hline Elsier & 1 \\
\hline Scielo & 3 \\
\hline Otros & 4 \\
\hline TOTAL & \\
\hline
\end{tabular}

Tabla 4. Distribución de los estudios con respecto al idioma de origen.

\begin{tabular}{cc}
\hline Fuentes o Base de datos & Número de Estudios \\
\hline Español & 22 \\
\hline Portugues & 1 \\
\hline
\end{tabular}

En la Tabla 4, se observa finalmente que el total de artículos incluidos fueron 22 en español y 1 en portugués.

Antes de entender cómo surge la adquisición y el desarrollo de los procesos lógicos se debe saber que pensar es un acto complejo que permite formar una serie de representaciones mentales para posteriormente obtener una acción, para conseguirlo se requiere de un conjunto de operaciones mentales como: identificación, ordenación, análisis, síntesis, comparación, abstracción, generalización, codificación, decodificación y clasificación entre otras, gracias a las cuales se pueden conformar estas habilidades del pensamiento denominadas pensamiento lógico matemático.

Estas a su vez son las que conducen al estudiante a asimilar los contenidos de las asignaturas, para que a continuación pueda utilizarlos en el momento que los requiera. Hoy día los sistemas educativos de muchos países han puesto gran énfasis en el aprendizaje de las matemáticas con la finalidad de mejorar los resultados en esta área de conocimiento, para ello Bryant y Nunes (2002) sugieren que la base del desarrollo matemático se basa en el pensamiento lógico, la enseñanza del sistema de numeración convencional y el aprendizaje significativo y contextualizado de los contenidos matemáticos, dicha enseñanza es impartida mediante una serie de enfoques, siendo el enfoque Piagetiano el que tiene mayor prevalencia en estos estudios (1).

A su vez es imprescindible considerar los aportes de la neurociencia, que como paradigma emergente, ha soportado, perfilado y en ocasiones, conceptualizado, al aprendizaje matemático, ya que sin la participación de la atención, la memoria de trabajo y las emociones no podría existir una relación entre aprendizaje, matemáticas y cerebro (2). 
Partiendo de la visión de las matemáticas, los niños de 0-3 años desarrollan nociones de ubicación espacial, de tiempo, reconocimiento de cualidades, de semejanzas y diferencias, relaciones entre objetos, agrupación, clasificación, ordenación, correspondencias y las primeras nociones de cantidad, continua y discreta. En consecuencia, las primeras nociones y relaciones que van a dar soporte a la construcción posterior de conocimientos matemáticos (3).

Las competencias lógico matemáticas iniciales van más allá de la adquisición de los principios subyacentes al conteo y la habilidad para determinar el cardinal de un conjunto, implican también la capacidad de establecer relaciones entre las cantidades en términos de adición, sustracción, multiplicación y división, habilidades que son desarrolladas a partir de los 4 años hasta los 6 aproximadamente (4). No existe una enseñanza de los procesos lógicos matemáticos, estos se adquieren a medida que experimentan el mundo, en otras palabras, se llevan a cabo a través de un proceso no formal.

En toda adquisición y/o desarrollo del pensamiento lógico matemático se proponen cinco estándares, así como también el manejo de tres tipos de pensamiento (numérico, espacial y métrico) (5) (6).

-La resolución de problemas, implica construir nuevo conocimiento matemático al reflexionar, aplicar y adaptar estrategias que favorecen la solución de situaciones problemáticas.

-El razonamiento y la demostración, que permite a los alumnos tomar mayor conciencia de que las matemáticas tienen sentido y ofrecen poderosas alternativas para lograr comprender una gran variedad de fenómenos.

-La comunicación, que en definitiva es una herramienta que promueve la interacción con otros para aclarar las ideas matemáticas; al fortalecer la comunicación, las ideas se transforman en objeto de reflexión, de precisión y discusión.

-Las conexiones, para enfatizar que las matemáticas no están constituidas por ejes temáticos desvinculados entre sí, sino que por el contrario, esta disciplina es un campo de estudio integrado.

-Las representaciones, que corresponden a las formas de representar las ideas matemáticas, las cuales pueden ser a través de imágenes, materiales concretos, tablas, gráficos, números, letras, entre otras.

Para determinar el estado de desarrollo de estos procesos se determinó la investigación sobre los métodos de evaluación, en donde se encontraron los siguientes:

Test de Evaluación Matemática Temprana de Utrecht (TEMTU) o The utrecht early mathematical competence test. (J.E.H. van Luit, B.A.M. van de Rijt \& A.H.Pennings): Evalúa el nivel de competencia temprana referente a conceptos de comparación, clasificación, correspondencia uno a uno, seriación, conteo verbal, conteo estructurado, conteo resultante y conocimiento general de los números. Esta prueba fue creada para niños de 4 a 7 años. (7)

Tema - 3 creado por Herbert P. Ginsburg, Arthur J. Baroody; adaptadores Maria Cristina Nuñez del Rio, Isabel Lozano Guerra: Evalúa las habilidades matemáticas de numeración, comparación de cantidades y las habilidades de cálculo informal, en niños de 3 y 8 años (8)

Examen lógico operativo de Feldman y su representación: Establece en qué nivel se encuentran las habilidades cognitivas del niño dependiendo de la clasificación de los estadios hechos por Piaget. Evalúa procesos de clasificación de objetos, figuras geométricas, seriación, alineamientos, relaciones asimétricas, cuantificadores, capacidad asociatividad, capacidad de abreviación, conservación del peso, conservación del volumen intuitivo, desligación volumen peso (9)

- Evamat - Prueba para la evaluación de la competencia matemática creado por Jesús García Vidal, Beatriz García Ortiz, Daniel Gonzalez Manjón, Eva M. Jimenez Mesa. Aplicado a niños de transición a $9^{\circ}$ grado. En cada edad evalúa los siguientes procesos (10):

- Evamat-0: Geometría, cantidad y conteo, resolución de problemas.

52 - Evamat-1: Numeración, cálculo, geometría, resolución de problemas.

Rev. Signos Fónicos Vol $3 \mathrm{~N}^{\circ} 1$

Abril / Septiembre 2017 
- Evamat-2: Numeración, cálculo, geometría, información y azar, resolución de problemas.

Existen varios test neuropsicológicos que contiene unos apartados que evalúan la aritmética como:

Test para el Diagnóstico de las Competencias Básicas en Matemáticas (TEDI-MATH) creado por J. Grégoire, M-P. Noël y C. Van Nieuwenhoven: Es una prueba que evalúa las destrezas matemáticas básicas en niños de 4 y 8 años. Dentro de los procesos evaluados está contar, numerar, comprensión del sistema numérico, operaciones lógicas, operaciones y estimación del tamaño. (11)

Protocolo neuropsicológico para la evaluación de las áreas instrumentales básicas: Evalúa los procesos cognitivos y las funciones básicas, frente a la necesidad de un diagnóstico precoz de las habilidades para la lectura y escritura, relacionadas con el aprendizaje escolar. Este manual está destinado para niños y niñas del último año de jardín que van a ingresar al primer año escolar, en el cual evalúa colores, ubicación espacial, relaciones espaciales, tamaños, cuantificadores, dimensiones, conceptos, figuras geométricas, conteo y numeración (12)

Evaluación del conocimiento matemático (Arthur Benton \& Alexander R. Luria): Evalúa la capacidad para comprender los números presentados en forma oral y escrita, el cálculo oral y escrito, contar series numéricas y elementos gráficos, y para el razonamiento matemático. Aplicado a niños entre $1^{\circ}$ y $6^{\circ}$ grado (13).

La evaluación de la competencia temprana en niños de 4 a 6 años, es imprescindible para determinar aquellas dificultades que puedan afectar en los años siguientes la educación matemática formal (14) (15)(1).

\section{ANÁLISIS Y DISCUSIÓN}

El desarrollo del pensamiento lógico, es un sumario de adquisición de códigos nuevos que hacen viable la comunicación con el ambiente, las relaciones lógico matemático componen la base necesaria para la adquisición de los conocimientos de todas las áreas académicas que dentro del futuro profesional de los niños y niñas; se habla que se constituye en un instrumento a través del cual se asegura la interacción humana, de allí la importancia del desarrollo de competencias de pensamiento lógico esenciales para la formación integral del ser humano.

El pensamiento lógico sirve entonces para analizar, argumentar, razonar, justificar o probar razonamientos, se caracteriza por ser preciso y exacto, basado en datos probables o hechos. El pensamiento lógico es analítico ya que divide los razonamientos en partes, racional puesto que, sigue reglas y a su vez secuencial ya que es lineal o va paso a paso.

Por otro lado, la matemática es la ciencia deductiva que se dedica al estudio de las propiedades de los entes abstractos y de sus relaciones. La matemática analiza las estructuras, magnitudes y vínculos de los entes abstractos mientras que el pensamiento lógico los aterriza y los centra en la razón.

De allí surge el pensamiento lógico matemático, que se constituye en una actividad cultural social e históricamente situada, influenciada por criterios prácticos de utilidad e intencionalidad basada en prácticas cotidianas como contar, medir, localizar, diseñar, jugar o explicar. Bishop (1999) (19).

En un contexto profesional e investigador el Currículo del Primer Ciclo de Educación Infantil según el Ministerio de Educación Nacional propone, entre otros, trabajar los siguientes contenidos:

1. Orientación de términos relativos al espacio (aquí, allá, dentro, fuera, arriba, abajo).

2. Orientación en las secuencias temporales (mañana, tarde, ahora, después, hoy, mañana).

3. Observación y actuación sobre la realidad inmediata, a partir de las propias vivencias, estableciendo relaciones entre objetos según sus características perceptivas. 
4. Observación y exploración del entorno físico y social, planificando y ordenando la propia acción, constatando los efectos y estableciendo relaciones entre la propia actuación y las consecuencias que se derivan.

5. Interés y curiosidad por el medio físico y social, explorando las características de objetos, materiales y elementos del entorno natural.

6. Iniciación en la diferenciación de algunas cualidades sensoriales. Inicio de las primeras clasificaciones, ordenaciones y correspondencias en función de las características y los atributos.

La adquisición de competencias necesarias para que se produzcan la comprensión del número está condicionada por el desarrollo previo, como los son los procesos cognitivos básicos (percepción, memoria, atención, razonamiento); conceptos básicos (forma, tamaño, cantidad, orden, posición), conocimiento de símbolos y signos necesarios para operar. De ahí parte la importancia del desarrollo de los pensamientos y habilidades matemáticas (20).

Por tanto es necesario dar a conocer de donde nacen los procesos del aprendizaje matemático, a continuación se destacarán los más importantes:

Teniendo en cuenta las etapas del desarrollo evolutivo denominadas por Jean Piaget, propuestas desde el constructivismo, a partir de las experiencias que le brinda la relación e interacción con los objetos que va construyendo el niño(a), nace la importancia de la segunda etapa piagetiana, en el que los niños realizan un salto cualitativo hacia delante gracias a su nueva habilidad para usar símbolos como las palabras para representar personas, lugares y objetos, esta etapa pre operacional que va de los 2 a los 7 años puede dividirse en dos sub-etapas: la función simbólica se presenta aproximadamente entre los dos y cuatro años, en esta sub-etapa, el niño adquiere la habilidad de representar mentalmente un objeto que no está presente. El pensamiento intuitivo inicia aproximadamente a los cuatro años de edad y termina alrededor de los siete años, en esta sub-etapa, los niños empiezan a utilizar un razonamiento primitivo y desean saber la respuesta a todo tipo de preguntas.

Hay, sin embargo, limitaciones importantes en el pensamiento; los niños generalmente no logran tener en cuenta todos los aspectos de una situación, de ahí nace la importancia de conocer los distintos subprocesos del pensamiento para el desarrollo lógico-matemático (21), Como primera instancia el pensamiento numérico se refiere a la comprensión que tiene una persona sobre los números y las operaciones junto con la habilidad para desarrollar estrategias útiles o manejar números u operaciones, el pensamiento espacial es considerado como el conjunto de los procesos cognitivos mediante los cuales se construyen y se manipulan las representaciones mentales de los objetos del espacio, la relaciones entre ellos, su transformación y sus diversas traducciones a representaciones materiales y por último el pensamiento métrico se refiere a la comprensión de magnitudes y cantidades y su uso en sentido y significado para la comprensión de situaciones en contextos (5).

Las primeras competencias matemáticas del niño se desarrollan a partir del aprendizaje informal, en otras palabras los niños preescolares manejan un previo conocimiento matemático, basado en experiencias cotidianas, por esta razón hablamos de los esquemas protocuantitativos, esquemas de razonamiento que permiten establecer juicios de cantidad sin atender a la numerosidad.

La integración de los esquemas protocuantitativos con el conteo dará al niño las competencias necesarias para enfrentarse a la resolución de situaciones problemáticas. Existen tres esquemas protocuantitativos, el primero es el de comparación son etiquetas lingüísticas usadas en la comparación de tamaños: mayor, menor, más, menos, más alto..., lo que permite hacer juicios de comparación sobre cantidades de material físico, el segundo esquema de incremento/decremento permite razonar sobre cambios en las cantidades cuando se les añade o quita algún elemento sin necesidad de ver los objetos en su estado anterior y posterior y el tercer esquema que habla de parte/todo permite al niño reconocer que cualquier "pieza" puede ser dividida en partes más pequeñas; que el "todo" es mayor que las "partes"; y que las partes se pueden recombinar para hacer el todo. Primer conocimiento de la propiedad aditiva de las cantidades (22).

\section{4}

Rev. Signos Fónicos Vol $3 \mathrm{~N}^{\circ} 1$

Abril / Septiembre 2017 
Antes de llegar a la adquisición del concepto de número, existe un período llamado pre numérico, cuyo objetivo fundamental es realizar actividades para que el niño vaya adquiriendo el concepto de conservación de las cantidades discontinuas, las nociones que se hallan en la base de la construcción del concepto de número son:

Conservación de cantidad y número: en tal sentido el número es una síntesis de dos aspectos: el cardinal y el ordinal que a su vez, tienen por base la síntesis de dos operaciones lógicas como la clasificación y la seriación. Dicha conservación implica la capacidad de percibir que una cantidad no varía.

La cardinalidad puede definirse como "la propiedad que tiene un conjunto con respecto a la totalidad de los elementos que lo forman, los números cardinales son utilizados para contar, para indicar una cantidad, pero no un orden.

La ordinalidad expresa la posición de algo en una lista, como primero, segundo, tercero, cuarto, quinto etc., por ejemplo cuando se colocan objetos en orden, se utilizan los números ordinales para nombrar suposición.

De acuerdo con la secuencia del desarrollo del pensamiento del niño, una vez que ha identificado las propiedades físicas de los objetos, deben establecer conexiones entre ellos por sus similitudes. Así se inicia la formación del concepto de clasificación.

La seriación se refiere a la relación entre objetos diferentes en cuanto a su dimensión, e implica una relación de orden, pues tiene la propiedad de la transitividad.

Partiendo del aprendizaje informal es como se desarrolla el pensamiento matemático, aprenden conceptos, ordenando y/o guardando juguetes o comestibles, adquieren las nociones de relaciones espaciales y de comparaciones de sólidos, construyendo con bloques, llevan a cabo representaciones, dibujan para grabar ideas elaboradas sobre rutinas diarias; aprenden términos direccionales entonando canciones acompañados de movimientos y de la visualización espacial (23).

Numerosos investigadores subrayan la importancia de trabajar las matemáticas en la infancia e insisten en que los profesores de educación infantil han de estar bien preparados para realizar este trabajo (24) (6).

En general, las investigaciones realizadas sobre las operaciones aritméticas elementales ponen de manifiesto que los niños, antes de recibir enseñanza formal sobre los algoritmos, construyen por sí mismos un amplio abanico de estrategias. Por ejemplo, Baroody y Tiilikainen (2003), a partir del modelo de elección de estrategias que se apoya en las teorías del procesamiento de la información y en la teoría de esquemas, han establecido seis estrategias de adición: (25) contar todo con objetos concretos (i.e., representan secuencialmente ambos sumandos y cuentan el total), (26) contar todo con objetos concretos utilizando atajos (i.e., representan simultáneamente con los dedos de cada mano, los sumandos y cuentan la cantidad representada), (20) conteo concreto de la cantidad añadida (i.e., representan indirectamente la cantidad inicial y directamente la cantidad añadida con objetos, de modo simultáneo o secuencial, antes de contar el total), (1) conteo abstracto (i.e., descubren que la representación de los dos sumandos puede hacerse simultáneamente con el recuento total, lo que requiere un proceso de registro de la cantidad añadida), (2) contar todo empezando por el sumando mayor (i.e., es semejante a la anterior, pero en ésta la suma se inicia por el sumando mayor) y (3) contar desde el sumando mayor (i.e., comienza el conteo con el cardinal que representa al sumando mayor y pro-siguen añadiendo el menor).

Igualmente, Baroody y Coslick (1998) encontraron las siguientes estrategias de sustracción: (1) quitar de (i.e., representan con objetos la cantidad correspondiente al minuendo y retiran de ese conjunto una cantidad de objetos equivalente al sustraendo), (2) añadir a (i.e., representan el sustraendo, añaden objetos hasta alcanzar el minuendo y cuentan la cantidad añadida) y (3) emparejamiento (i.e., representan el minuendo e inmediatamente debajo el sustraendo y finalmente, cuentan los objetos que no están emparejados). 
Pero las competencias matemáticas iniciales van más allá de la adquisición de los principios subyacentes al conteo y la habilidad para determinar el cardinal de un conjunto, implican también la capacidad de establecer relaciones entre las cantidades en términos de adición, sustracción, multiplicación y división (Alibali, 1999).

Los estudios sobre las estrategias de multiplicación y división de 4 a 6 años guardan ciertos paralelismos con los de adición y sustracción. En concreto, revelan que los niños pequeños recurren a estrategias informales como: (1) el recuento unitario (p.e., en un problema de multiplicación, hacen grupos de " $x$ " elementos y cuentan todos los objetos), (2) el doble recuento (i.e., integran dos secuencias de conteo; por ejemplo, en un problema de división, generan la secuencia de números correspondiente al dividendo, de forma simultánea llevan la cuenta de los grupos que forman según el tamaño del divisor, siendo la respuesta el número de grupos contados) y (3) el conteo de transición (i.e., calculan la respuesta utilizando una secuencia de conteo basada en múltiplos de un mismo factor) (p.e., Anghileri, 1999; Caballero, 2006; Carpenter, Fennema, Fran-ke, Levi y Empson, 1999; Wright, Mulligan y Gould, 2000).

Dicha enseñanza se construye en el pensamiento a medida que se estructura lógicamente la realidad, a partir de la interacción con el entorno. Estas concepciones piagetianas insisten en la importancia de las operaciones lógicas para construir los conceptos numéricos y aritméticos. Por ello la acción docente debería centrarse en la mediación para la construcción de las nociones lógico-matemáticas y en los aspectos lógicos subyacentes. Igualmente, los procedimientos mecánicos y memorísticos, tan frecuentes en las aulas, deberían suprimirse a su mínima presencia, a favor de la comprensión de tales nociones y su aplicación práctica (27)

Es indudable que las neurociencias jueguen un papel importante en las ciencias de la educación matemática. Para Luria (1988), la atención ocupa un papel fundamental, el tallo cerebral y el sistema activador reticular ascendente, denominado por las siglas SARA, son los que controlan los estados de alerta y vigilia necesarios para que se active dicha función atencional, la memoria de trabajo asume también un papel relevante para el cálculo y predice que el entrenamiento activo de los lóbulos frontales mejora sustancialmente esta capacidad.

Por último, las emociones son inherentes al ser humano y muchas veces se manifiestan como una combinación letal para el aprendizaje. Un aspecto que se destaca en estas investigaciones es el referido a las dos estructuras del sistema límbico: la amígdala y el hipocampo. Estas estructuras están relacionadas directamente con la corteza neo frontal. Si por alguna razón resultan inhibidas a causa del miedo o alguna situación de estrés en el aprendizaje de las matemáticas, puede existir una disminución en la capacidad de atención y motivación, afectando también el rendimiento. Es por ello, que el objetivo fundamental de las pruebas matemáticas consiste en identificar las alteraciones del desarrollo numérico que puede presentar el niño.

En relación a la búsqueda de pruebas utilizadas para evaluar el desarrollo lógico matemático en niños de la primera infancia, se encuentra el Utrech Early Numeracy Test, creado por Johannes van Luit, Bernadette van de Rijt y Albér Pennings en 1994, que ha sido adaptado y estandarizado en España bajo el nombre de Test de Evaluación Matemática Temprana de Utrech, el cual contiene las características psicométricas para evaluar las competencias de los niños de 4 - 7 años. Consta de 3 versiones paralelas ( $A, B$ y $C$ ) de 40 ítems cada una. Se compone de 8 subtes y cada uno tiene cinco ítems. Los 8 subtest reúnen actividades relacionadas con las operaciones piagetianas y tareas de conteo.

Los primeros 4 subtest evalúan habilidades de tipo piagetiano (conceptos de comparación, clasificación, correspondencia uno a uno, seriación) y los otros cuatro habilidades numéricas de corte cognitivo (conteo verbal, conteo estructurado, conteo resultante y conocimiento general de los números) (7). 


\begin{tabular}{|c|c|}
\hline Conceptos de comparación & $\begin{array}{l}\text { «Mira estos edificios. Señala el edificio más } \\
\text { pequeño». }\end{array}$ \\
\hline Clasificación & $\begin{array}{l}\text { * ¿Puedes señalar el que tiene cinco cuadrados } \\
\text { pero ningún triángulo?». }\end{array}$ \\
\hline Correspondencia uno a uno & $\begin{array}{l}\text { El evaluador le da al niño } 15 \text { cubos y le presenta } \\
\text { un dibujo que representa las caras de dos dados } \\
\text { con el patrón de puntos de } 5 \text { y } 6 \text {. «Yo he lanzado } \\
\text { dos dados y he conseguido estos puntos. } \\
\text { ¿Puedes darme la misma cantidad de cubos?». }\end{array}$ \\
\hline Seriación & $\begin{array}{l}\text { «Aquí ves unos cuadrados que tienen unos } \\
\text { palitos. Señala el cuadrado donde los palitos } \\
\text { están ordenados del más delgado al más grueso». }\end{array}$ \\
\hline Conteo Verbal & $\begin{array}{l}\text { En este subtest se evalúa la secuencia numérica } \\
\text { oral hasta el } 20 \text {. La puede contar hacia adelante, } \\
\text { hacia atrás. }\end{array}$ \\
\hline Conteo estructurado & $\begin{array}{l}\text { El evaluador pone sobre la mesa un total de } 20 \\
\text { cubos de forma desorganizada. El niño es } \\
\text { requerido a que cuente todos los cubos. Se le } \\
\text { permite señalar o mover los cubos. }\end{array}$ \\
\hline Conteo resultante (sin señalar) & $\begin{array}{l}\text { Se le presenta al niño } 15 \text { cubos en tres filas de } \\
\text { cinco cada una con un espacio entre ellos y se le } \\
\text { pregunta: « ¿Cuántos cubos hay aquí?». }\end{array}$ \\
\hline Conocimiento general de los números & $\begin{array}{l}\text { «Tú tienes } 9 \text { canicas. Pierdes } 3 \text { canicas. ¿Cuántas } \\
\text { canicas te quedan?». }\end{array}$ \\
\hline
\end{tabular}

Esta prueba ha sido adaptada en diferentes países, siendo Chile uno de ellos donde los resultados expresan dificultades en cada ítem propuesto, obteniendo puntajes altos en los ítems de comparación y clasificación, lo contrario a los de conteo verbal y conteo estructurado, lo cual se debe a que el currículo de la educación actual se empeña en actividades de tipo piagetiano, considerándose un prerrequisito para el desarrollo del número en el niño (1).

Además, el tema - 3 está compuesto por 72 ítems que valoran diferentes aspectos de la competencia matemática básica. Los aspectos informales de la matemática (actividades que no precisan el uso de símbolos escritos) son valorados mediante 41 ítem, que pueden repartirse en cuatro categorías: a) numeración, b) comparación de cantidades, c) habilidades de cálculo informal, d) conceptos. La escala formal (actividades que implican el uso de los símbolos matemáticos, está compuesta por 31 ítems, distribuidos en 4 componentes: a) conocimiento de convencionalismos, b) hechos numéricos, c) habilidades de cálculo, d) componentes de base 10 (28).

Por otro lado, el test de Feldman consta de 11 pruebas, en las que engloba varias de las mencionadas en los dos test anteriores. En la clasificación de objetos busca valorar el nivel de conceptualización y de abstracción del niño, la clasificación de figuras geométricas está dirigida a establecer la agilidad conceptual, en la seriación valora la capacidad del niño de ordenar ciertos objetos en $\mathbf{5} 7$ 
orden decreciente, a su vez comprenda que es injertar y corregir en el caso de que falle en una o dos de las tres maniobras presentadas en este apartado.

Así mismo, en la prueba de alineamientos, tiene 3 momentos: correspondencia de tipo provocada y/o espontánea, exclusión y conservación que consiste básicamente en la cardinalidad del número; en las relaciones asimétricas estima las comparaciones de pares opuestos de cualidades donde se manipula indirectamente nociones de "mucho-poco"; los cuantificadores tienen las mismas condiciones que las relaciones anteriores pero se refieren a cantidades no numéricas específicamente; en la capacidad de asociatividad y abreviación valora si el niño comprende las consignas dadas; en las pruebas de conservación de peso y conservación de volumen intuitivo el niño debe dar una respuesta verbal ante lo sucedido en cada una de las maniobras realizadas, estas dos pruebas son parecidas por lo que no se recomienda realizarlas el mismo día puesto que el niño se condicionará y dará las mismas respuestas en las dos; finalmente en la deligación volumen-peso se valora la capacidad del niño para identificar la diferencia de los materiales empleados (9).

Por otra parte, se encuentra el Evamat que evalúa diferentes procesos en cada año escolar, en el grado de transición y primero, contienen pruebas de geometría donde se da la consigna de marcar en cada recuadro la figura mencionada, cantidad y conteo y resolución de problemas.

En el grado de primero y segundo primaria, se presentan pruebas de numeración de diferentes dibujos bajo unas consignas relacionadas a sus características como tamaño, peso, altura y cantidad; en las pruebas de cálculo incluye sumas-restas, cálculo mental, descomposición aditiva de los números, seleccionar el menor de los números, descomponer en decenas y unidades, utilización de números ordinales; la prueba de geometría consiste en marcar diferentes figuras, buscar y contar figuras, identificación de figuras, representación de posiciones, identificación de la figura resultante; en la resolución de problemas se basan en contar y representar objetos, contar figuras y completar una tabla, unir la palabra con una operación y problemas.

Finalmente, en el grado de segundo y tercero de primaria, las pruebas de numeración consisten en continuar las series, escribir los números antecesor y sucesor, escribir el número mayor y el menor de cada grupo, marcar números impares, ordenar números de manera creciente, completar centenas, decenas y unidades; dentro de la prueba de cálculo está relación de multiplicaciones, calcular mentalmente unas operaciones, resolución de operaciones y aproximación de números, en la prueba de geometría trabaja unidades de medida y atributos; por último se encuentran las pruebas de información y azar y la resolución de problemas (10).

Tras la búsqueda realizada se pudo evidenciar la escasez de instrumentos para la evaluación de las matemáticas en educación infantil, debido a que se encuentran más para niños en edades de 8 a 15 años. Sin embargo, existen unos test neuropsicológicos que contienen unos subtest que evalúan la aritmética como se mencionó anteriormente en el Test para el Diagnóstico de las Competencias Básicas en Matemáticas (TEDI-MATH) el cual contiene 25 pruebas diferentes agrupadas en 6 grandes ámbitos de conocimiento numérico como contar, numerar, comprensión del sistema numérico, operaciones lógicas, operaciones aritméticas y estimación del tamaño (11). Este tipo de prueba además de valorar las competencias matemáticas permite identificar la estrategia que maneja el niño para dar respuesta a las tareas planteadas.

Así mismo, el Protocolo neuropsicológico para la evaluación de las áreas instrumentales básicas valora colores, ubicación espacial, relaciones espaciales, tamaños, cuantificadores, dimensiones, conceptos, figuras geométricas, conteo y numeración al igual que las pruebas matemáticas mencionadas anteriormente (12). Además, la evaluación del conocimiento matemático propuesto por Benton y Luria está compuesta por 8 subtest que se basan en la valoración cuantitativa de números presentados visualmente, escritura de números al dictado, copia de números, cálculo oral y escrito, conteo de elementos gráficos uno a uno y en agrupaciones, conteo de series numéricas y resolución de problemas(13).

\section{8}

Rev. Signos Fónicos Vol $3 \mathrm{~N}^{\circ} 1$

Abril / Septiembre 2017 
La propuesta de protocolo de evaluación de procesos lógicos- matemáticos realizada por el equipo investigador surge ante la necesidad de instrumentos que evalúen las competencias lógico-matemáticas en edad temprana (29). Para elaborarla se llevó a cabo un análisis de la revisión de la literatura científica sobre el desarrollo evolutivo lógico-matemático, pruebas de evaluación halladas y parámetros para la elaboración de un protocolo de evaluación en matemáticas temprana. Este instrumento se diseña para niños en edades de 4-6 años. Para Piaget estas edades están dentro del Período pre operacional (de 2 a 7 años), en este periodo suceden grandes cambios en la construcción intelectual en el niño, lo cual hay que aprovechar y tener en cuenta en su formación. El niño aquí presenta un razonamiento de carácter intuitivo y parcial, razona a partir de lo que ve, por ello domina en él la percepción (función básica superior que determina el imaginario). Se pueden considerar en este período dos etapas: pre-conceptual e intuitivo (28), esta última etapa especialmente de nuestro interés, ya que comprende las edades de 4 a 7 , abarcando las edades seleccionadas para esta prueba. En esta etapa el pensamiento está dominado por las percepciones inmediatas. Sus esquemas siguen dependiendo de sus experiencias personales y de su control perceptivo. Son esquemas pre- lógicos.

Dada la importancia de estos primeros acercamientos y apoyándonos en Gardner (2000), quien desde una perspectiva neuropsicológica afirma que las distintas etapas del desarrollo corresponden con unos patrones neurológicos distintos y que en esta relación cerebro-mente, las experiencias de principio de la vida tienen una importancia especial para la vida posterior (1), se justifica el interés por el conocimiento de la adquisición y desarrollo de los procesos lógico-matemáticos de los niños en edad temprana, ya que resulta el momento adecuado para evaluar y así identificar debilidades y fortalezas que puedan dar luz a estrategias educativas eficaces que apunten a mejorar los procesos de aprendizaje (30).

El pensamiento constructivista es un conjunto de posturas de tipo epistemológico y pedagógico, frente a la realidad natural, humana y social. Una de las posturas constructivistas más relevantes es el constructivismo piagetiano, esta postura tiene como referente los postulados de la epistemología genética, donde se origina el entramado teórico piagetiano, los cuales son la teoría de las etapas, la teoría de la equilibración, la utilización de los esquemas de razonamiento formal y el posicionamiento epistemológico (31).

Vale la pena aclarar que una de las principales tesis del constructivismo afirma que en el estímulo y la respuesta dada por el sujeto de aprendizaje se producen procesos intermedios, mediante los cuales los sujetos interpretan el estímulo, poniendo en él aspectos que no se encuentran en el dato original, lo cual implica que el conocimiento es una construcción subjetiva (32). Por esa razón, en sus estudios Piaget examinó cómo se forman los conocimientos y cómo cambian, cuáles son las etapas que tienen lugar en el proceso de adquisición del conocimiento y, además, las formas como se organizan los mismos, lo cual le permitió concluir que las formas que sirven para organizar el conocimiento no son innatas, sino que se van adquiriendo a lo largo de la vida.

Por tanto, la construcción de conceptos matemáticos apoyada en un enfoque constructivista, implica por lo menos las siguientes acciones (33):

- Partir del nivel de desarrollo del estudiante y de sus preconceptos.

- Crear entornos de aprendizaje significativo.

- Propiciar el desarrollo de la inteligencia lógico-matemática mediante el diseño de actividades lúdicas que permitan utilizar los conocimientos matemáticos y la capacidad de razonamiento en un ambiente creativo y recreativo.

- Dar oportunidad para que los estudiantes realicen aprendizajes significativos por sí solos y en equipo.

- Contextualizar los procesos de construcción del conocimiento, de tal forma que los estudiantes modifiquen los esquemas que ya poseen.

- Establecer relaciones entre el nuevo conocimiento y el conocimiento ya existente, relacionando los distintos pensamientos matemáticos.

- Analizar y solucionar situaciones problema y/o problemas. 
En numerosas investigaciones en el proceso lógico matemático se nombran las competencias matemáticas iniciales como pre recurrentes para la adquisición y desarrollo de las matemáticas (10) (7) (1) (8) (28), pero es poca evidencia de la importancia del desarrollo del pensamiento matemático a través de la adquisición por etapas para la adquisición y desarrollo del mismo (34) (35), existiendo cuatro tipos de este: pensamiento numérico, pensamiento espacial, pensamiento métrico y pensamiento aleatorio. Siendo los tres primeros manifestados durante los primeros años de vida.

Lo cierto, es que, se evidencio que las competencias matemáticas iniciales están inmersas dentro de esos tres tipos de pensamientos matemáticos (34), siendo así:

- $\quad$ En el pensamiento numérico se incluyen conceptos matemáticos como: cuantificadores, conteo automático, correspondencia uno a uno, seriación y operaciones básicas. Contribuyendo así el desarrollo de la comprensión numérica y la operacional.

- $\quad$ El pensamiento espacial abarca conceptos matemáticos de: discriminación de formas, copia de modelos, ubicación espacial y relaciones espaciales. Contribuyendo a la adquisición de las representaciones mentales de los objetos del espacio.

- $\quad$ Dentro del pensamiento métrico se hayan conceptos matemáticos referentes a dimensiones de: longitud, peso y tiempo. Contribuyendo al desarrollo de la comprensión de magnitudes y cantidades, su utilidad, y su función.

En las pruebas de evaluación de la matemática temprana se halló que únicamente abarcan la evaluación de las competencias matemáticas iniciales (7)(28)(1)(8)(10)(13)(9) (operaciones piagetianas) pero para este instrumento de evaluación proponemos la integración de los pensamientos matemáticos con estas competencias matemáticas.

\section{CONCLUSIONES}

De acuerdo al enfoque piagetiano el mundo físico y social en el que se desarrollan los niños pequeños les brinda la oportunidad de adquirir numerosos conceptos relacionados con la aritmética, antes de la enseñanza formal. En los aportes anteriores se indica que los contenidos educativos deberían trabajarse desde una visión integral que incluya procesos, enfoques y actividad cerebral con el fin de favorecer su uso comprensivo y eficaz en diferentes contextos.

La finalidad de las pruebas evaluativas es identificar qué niños y niñas en Educación Infantil presentan alteraciones significativas en los componentes del sentido numérico, se sugiere la estructuración y aplicación de actividades de refuerzo, como también la consolidación y mantenimiento de aquellos aspectos destacados como normales para su edad. Es importante la valoración de la competencia matemática en un estadio temprano, ya que resulta el momento adecuado para identificar debilidades y fortalezas que puedan dar luz a estrategias eficaces que apunten a mejorar los procesos de aprendizaje.

La articulación de las competencias matemáticas iniciales con los tipos de pensamiento matemático posibilita la construcción de un instrumento integral y una visión holística del desarrollo lógico-matemático. Finalmente es necesario realizar la prueba piloto del protocolo de evaluación de habilidades lógico- matemáticas propuestas para dar continuidad al proceso de validación que se pretende realizar.

\section{REFERENCIAS}

1. Navarro Guzmán, J. I., Aguilar Villagrán, M., Marchena Consejero, E., Alcalde Cuevas, C., \& García Gallardo, J. Evaluación del conocimiento matemático temprano en una muestra de $3^{\circ}$ grado de educación infantil. Revista de educación. 2010;(601-615).

2. E M. Aportes de las neurociencias para el desarrollo de estrategias de enseñanza y apren- dizaje de las matemáticas.. Electrónica educare. 2010.

3. MEi. B. Ahí empieza todo. Las matemáticas de cero a tres años. Revista de didácticas de

60 las matemáticas. 2012. 
4. Rodríguez P, Oliva Lago , Caballero , Dopico C, Jiménez L, Solbes I.. El desarrollo de las estra- tegias infantiles. Un estudio sobre el razonamiento aditivo y multiplicativo. Anales de psicología. 2010.

5. AM VR. Desarrollo del pensamiento lógico-matemático. 2013.

6. Coronata AA. Hacia la alfabetización numérica en Educación Infantil: algunos avances en Chile y España. Educación matemática en la infancia. 2012;(42-56).

7. Cerda Etchepare, Pérez Wilson C, Moreno Araya C, Núñez Risco K, Quezada Herrera, Rebolledo Rojas J. Adaptación de la versión española del Test de Evaluación Matemática Temprana de Utrecht en Chile. Estudios pedagógicos. 2012.

8. García Vidal J, García Ortiz B, González Manjón D, Jiménez Mesa EM. Evamat - Prueba para la evaluación de la competencia matemática Santiago de Chile: EOS. .

9. Aragón EL, Delgado CI, Aguilar, Araújo A, Navarro JI. Estudio de la influencia de la inteli- gencia y el género en la evaluación matemática temprana. European Journal of Education and Psychology. 2013.

10. Arias RdIdAap. 2003..

11. Benton LA. Evaluación del conocimiento matemático. .

12. Peri LAV. Test de operaciones básicas para el aprendizaje de las matemáticas. Revista de investigación en psicología. 2007.

13. Estíbaliz Aragón Mendizábal, Manuel Aguilar Villagrán, José Ignacio Navarro Guzmán. Sis- tema Instruccional de apoyo a la enseñanza del sentido numérico. Revista de Educación. 2017.

14. Chacón IMG. Los protocolos de resolución en la enseñanza de matemáticas. 2013.

15. Gallego CAM, Redondo AL, Cabeza JAM, Gil DMD, Carvajal SMY, Clavijo ZA, et al. DISCAPACIDAD: UNA PERSPECTIVA DESDE LOS EJES DE DESIGUALDAD. Rev CIENTÍFICA SIGNOS FÓNICOS [Internet]. 2016 Oct 25 [cited 2016 Nov 15];2(3). Available from: http://revistas.unipamplona.edu.co/ojs_viceinves/index.php/CDH/article/view/2016

16. Cupani M. Validación de una nueva escala de expectativas de resultados y metas de ren- dimiento para matemática. Interdisciplinaria. 2010.

17. Bustamante C. El desarrollo de la noción de espacio en el niño de educación inicial. Ac- ción pedagógica. 2004; 1-9.

18. J P. El lenguaje y el pensamiento del niño. PAIDOS IBERICA. 1984.

19. Trevisan AL AR. A taxonomia revisada de vloom aplicada a avaliacao: um estudio de pro- vas escritas de matemáticas. Scielo. 2016; 451-464.

20. Bermejo V, Lago O. Aprendiendo a contar. Su relevancia en la comprensión y fundamen- tación de los primeros conceptos matemáticos. C.I.D.E.. 2007.

21. Páez C. Piaget; lenguaje conocimiento y educación. Revista Colombiana en Educación. 2011.

22. Castro E, Cañadas, Castro Rodríguez E. Pensamiento numérico en edades tempranas. Educación matemática en la infancia. 2013.

23. CARDOSO ESPINOSA, CERECEDO MERCADO MT. El desarrollo de las competencias matemáticas en la primera infancia. Iberoamericana en educación. 2008.

24. D RM. Las estrategias didácticas en la construcción de las nociones lógico-matemáticas en la educación inicial. Paradigma. 2009.

25. Salgado Somoza M, Salinas Portugal MJ. Competencia Matemática en niños de 4 años. Educación matemática en la infancia. 2012.

26. Portilla EMP, Claro DAB, Rodriguez SCP, Castro YMP, Carreño LGL, Ramirez MMR, et al. ANALISIS Y VALIDACION METODOLOGICA PARA EL ESTUDIO DE LOS DETERMINANTES SOCIALES EN SALUD Y LA DISCAPACIDAD. Rev CIENTÍFICA SIGNOS FÓNICOS [Internet]. $2016 \mathrm{Apr} 1$ [cited 2016 Nov 15];2(1). Available from: http://revistas.unipamplona.edu.co/ojs_viceinves/index.php/CDH/article/view/1838

27. Calvo GS. La evaluación de las matemáticas en Educación Primaria. Universidad de la Rioja. 2014.

28. Portilla EMP, Murillo EAA, Espinel DEB, Moncada LB, Parra IYM, Acevedo SSG, et al. EDUCACION PARA LA DIVERSIDAD. Rev CIENTÍFICA SIGNOS FÓNICOS [Internet]. 2015 Oct 1 [cited 2016 Nov 15];1(3). Available from: http://revistas.unipamplona.edu.co/ojs_viceinves/index.php/CDH/article/view/1570

29. Carlos Humberto Barreto Tovar, Luis Fernando Gutiérrez Amador, Blanca Ligia Pinilla Díaz, Ciro Parra Moreno. Límites del constructivismo pedagógico. Educación y educadores. 2006 Junio; 9(1).

30. Carretero M. Constructivismo y Educación. 2009 octubre.

31. AMR. V. DESARROLLO DEL PENSAMIENTO LÓGICO-MATEMÁTICO. BASADO EN LA TAXONOMIA DE BLOOM. 2013.. AM. M. Desarrollo matemático temprano. 2016. 